\title{
MAXIMAL FIELDS DISJOINT FROM FINITE SETS
}

W. R. SCOTT

Let $F$ be a subfield of an algebraically closed field $A$ of characteristic $0, S$ a finite subset of $A$ disjoint from $F, K$ a subfield of $A$ containing $F$ and maximal with respect to disjointness from $S, L$ a finite extension of $K$, and $G=G(L / K)$ the group of automorphisms of $L / K$. Quigley [4] and McCarthy [1] obtained precise information about $G$ in the case where $S$ has one or two elements, respectively (they handled the characteristic $p$ case also). Theorem 1 of this paper shows that there is some restriction on $G$ in the general case. In particular (Theorem 2), $G$ is solvable if $S$ has at most twenty elements.

Lemma. If $r$ is a positive integer, then there is a finite set II of primes such that if $G$ is a finite group containing at most $r$ maximal subgroups, then either

(i) G is cyclic of order $p_{1}^{n_{1}} \cdots p_{k}^{n_{k}}, p_{i}$ prime, $k \leqq r$, or

(ii) $G$ is a II-group.

Proor. Let $G$ contain at most $r$ maximal subgroups $H_{i}$, and let the index of $H_{i_{1}} \cap \cdots \cap H_{i_{s}}$ in $G$ be denoted by $n_{i_{1}} \ldots i_{s}$. If $G$ is cyclic, then conclusion (i) clearly holds. Assume that $G$ is not cyclic. We may count elements as follows:

$$
\begin{aligned}
o(G)= & {\left[o\left(H_{1}\right)\right]+\left[o\left(H_{2}\right)-o\left(H_{1} \cap H_{2}\right)\right] } \\
& +\left[o\left(H_{3}\right)-o\left(H_{1} \cap H_{3}\right)-o\left(H_{2} \cap H_{3}\right)\right. \\
& \left.\quad+o\left(H_{1} \cap H_{2} \cap H_{3}\right)\right]+\cdots
\end{aligned}
$$

Dividing by $o(G)$, we get

$$
\begin{aligned}
1= & 1 / n_{1}+\left(1 / n_{2}-1 / n_{12}\right) \\
& +\left(1 / n_{3}-1 / n_{13}-1 / n_{23}+1 / n_{123}\right)+\cdots .
\end{aligned}
$$

We may take $n_{1} \leqq n_{2} \leqq \ldots$. Also each expression surrounded by parentheses is positive since the $H_{i}$ are distinct maximal subgroups. Moreover, each such expression is less than or equal to its first term. It follows readily that $n_{1} \leqq r$. Suppose inductively that $n_{i_{1}} \cdots n_{i_{s}}$ have all been chosen for $i_{1}<\ldots<i_{s}<t$. The corresponding sum equals $1-a$, say. Then $n_{t} \leqq r / a$, and $n_{i_{1} \cdots t} \leqq n_{i_{1}} \cdots n_{t}$. Thus, by induction, there are only a finite number of choices for the $n_{i j k} \ldots$.

Received by the editors July 16, 1967 . 
Now the intersection $\Phi(G)$ of all $H_{i}$ is normal, and there are only a finite number of possibilities for $o(G / \Phi(G))$. Let $\Pi$ be the set of primes dividing such orders. By a theorem of Suzuki [5, p. 347], if $p \mid o(G)$ then $p \mid o(G / \Phi(G))$. Hence $G$ is a $\Pi$-group.

THEOREM 1. Let $r$ be a positive integer and $\mathrm{II}$ the set of primes guaranteed by the lemma. Let $F$ be a field of characteristic $0, A$ an algebraically closed field containing $F, S$ a subset of $A$ disjoint from $F$ and containing exactly $r$ elements, and $K$ a subfield maximal such that $K \cap S=\varnothing$ and $F \subset K \subset A$. Then there is a set $S^{\prime}$ of $r$ or fewer primes such that, if $L$ is a finite extension of $K$, then $G(L / K)$ is either

(i) cyclic of order $p_{1}^{n_{1}} \cdots p_{k}^{n_{k}}, p_{i} \in \Pi \cup S^{\prime}, k \leqq r$, or

(ii) a II-group.

Proof. $K$ exists by Zorn. By [1, Lemma 1$], A / K$ is algebraic. The field $K$ has at most $r$ minimal extensions in $A$, for each such extension contains an element of $S$. Let $M$ be the smallest subfield of $A$ which is normal over $K$ and which contains $L$ and these minimal extensions. Then $M$ is finite Galois over $K . G(M / K)$ has at most $r$ maximal subgroups by the fundamental theorem of Galois theory. By the lemma, $G(M / K)$ is cyclic of order $p_{1}^{n_{1}} \cdots p_{\boldsymbol{k}}^{n_{k}}, k \leqq r$, or a $\Pi$-group. Let $S^{\prime}$ be the set of all $p_{i} \notin \Pi$ that occur as $L$ varies. If $S^{\prime}$ contains distinct primes $p_{1}, \cdots, p_{r+1}$, then there are finite Galois extensions $M_{i}$ such that $p_{i} \mid o\left(G\left(M_{i} / K\right)\right)$. The composite of the $M_{i}$ is then a finite Galois extension $M$ such that either $G(M / K)$ is cyclic with order divisible by more than $r$ primes, or is noncyclic and not a $\Pi$-group. In either case this is a contradiction. Hence the theorem is true for $L$ replaced by $M$. In case (i), it is clear that $G(L / K)$ also has the form (i). In the other case, we use the easily proved result that ${ }^{1}$

$$
G(L / K) \cong N(G(M / L)) / G(M / L)
$$

where the normalizer is taken in $G(M / K)$. Since subgroups and factor groups of $\Pi$-groups are II-groups, $G(L / K)$ is a II-group.

For small values of $r$, the set $\Pi$ in the lemma and Theorem 1 may be determined from known theorems. In fact, it follows from [2] that for $r=1,2,3,4$, the set $\Pi$ may be taken as $\varnothing, \varnothing,\{2\}$, and $\{2,3\}$, respectively.

Pazderski [3] has shown that if a finite group $G$ contains fewer than 21 maximal subgroups, then $G$ is solvable. Hence we have

Theorem 2. If, in Theorem $1, r<21$, then $G(L / K)$ is solvable.

1 This fact was pointed out to me by Robert Gordon. 


\section{BIBLIOGRAPHY}

1. P. J. McCarthy, Maximal fields disjoint from certain sets, Proc. Amer. Math. Soc. 18 (1967), 347-351.

2. G. A. Miller, Maximal subgroups of a finite group, Proc. Nat. Acad. Sci. U. S. A. 27 (1941), 212-214.

3. Gerhard Pazderski, Über maximale Untergruppen endlicher Gruppen, Math. Nachr. 26 (1963/1964), 307-319.

4. Frank Quigley, Maximal subfields of an algebraically closed field not containing a given element, Proc. Amer. Math. Soc. 13 (1962), 562-566.

5. M. Suzuki, On the lattice of subgroups of finite groups, Trans. Amer. Math. Soc. 70 (1951), 345-371.

UNIVERSITY OF UTAH 